\title{
Resveratrol sensitizes glioblastoma-initiating cells to temozolomide by inducing cell apoptosis and promoting differentiation
}

\author{
HAO $\mathrm{LI}^{1,2}$, YAODONG LIU ${ }^{1,2}$, YUMIN JIAO ${ }^{1,2}$, ANCHEN GUO ${ }^{2,3}$, XIAOXUE XU $^{4}$, \\ XIANJUN QU ${ }^{5}$, SHUO WANG ${ }^{1,2}$, JIZONG ZHAO ${ }^{1,2} \mathrm{YE} \mathrm{LI}^{5}$ and $\mathrm{YONG} \mathrm{CAO}^{1,2}$ \\ ${ }^{1}$ Department of Neurosurgery, Beijing Tiantan Hospital, Capital Medical University, Beijing 100050; \\ ${ }^{2}$ China National Clinical Research Center for Neurological Diseases, Beijing 100050; ${ }^{3}$ Laboratory of \\ Clinical Medicine Research, Beijing Tiantan Hospital, Capital Medical University, Beijing 100050; \\ ${ }^{4}$ Medical Experiments and Testing Center, Capital Medical University, Beijing 100069; ${ }^{5}$ Department of Pharmacology, \\ School of Chemical Biology and Pharmaceutical Sciences, Capital Medical University, Beijing 100069, P.R. China
}

Received July 4, 2015; Accepted August 20, 2015

DOI: 10.3892/or.2015.4346

\begin{abstract}
Glioblastoma-initiating cells play crucial roles in the origin, growth, and recurrence of glioblastoma multiforme. The elimination of glioblastoma-initiating cells is believed to be a key strategy for achieving long-term survival of glioblastoma patients due to the highly resistant property of glioblastoma-initiating cells to temozolomide. Resveratrol, a naturally occurring polyphenol, has been widely studied as a promising candidate for cancer prevention and treatment. Whether resveratrol could enhance the sensitivity of glioblastoma-initiating cells to temozolomide therapy has not yet been reported. Here, using patient-derived glioblastomainitiating cell lines, we found that resveratrol sensitized glioblastoma-initiating cells to temozolomide both in vitro and in vivo. Furthermore, we showed that resveratrol enhanced glioblastoma-initiating cells to temozolomide-induced apoptosis through DNA double-stranded breaks/pATM/pATR/p53 pathway activation, and promoted glioblastoma-initiating cell differentiation involving p-STAT3 inactivation. Our results propose that temozolomide and resveratrol combination strategy may be effective in the management of glioblastoma patients, particularly for those patients who have been present with a high abundance of glioblastoma-initiating cells in their tumors and show slight responsiveness to temozolomide.
\end{abstract}

Correspondence to: Dr Yong Cao, Department of Neurosurgery, Beijing Tiantan Hospital, Capital Medical University, 6 Tiantanxili, Beijing 100050, P.R. China

E-mail: caoyong6@hotmail.com

Dr Ye Li, Department of Pharmacology, School of Chemical Biology and Pharmaceutical Sciences, Capital Medical University, 10 Xitoutiao, Youanmenwai, Beijing 100069, P.R. China

E-mail: yli@ccmu.edu.cn

Key words: glioblastoma-initiating cells, resveratrol, temozolomide, chemoresistance, combination

\section{Introduction}

Glioblastoma (GBM) is the most frequent primary brain tumor in adults and presents a very aggressive course with few therapeutic options. Recent studies have shown that GBM is composed of cell populations that are heterogeneous in terms of morphology and differentiation status (1). It has been proposed that a small population of tumor cells, named glioblastomainitiating cells (GICs), plays a crucial role in the origin, growth, recurrence and drug resistance of GBM (2). Studies suggest that the limited therapeutic efficacy of conventional approaches to GBM is due to the resistant nature of GICs to chemotherapy and radiotherapy, allowing the survival of GICs to regenerate the tumor (3). GICs are thus considered the major barrier to GBM therapy, and elimination of GICs is considered to be a key strategy for achieving the long-term survival of GBM patients $(1,4)$. At present, temozolomide (TMZ) is the standard chemotherapy drug for GBM, yet significant GICs resistance toward TMZ has been widely reported $(5,6)$. Recent findings have even noted the significant expansion of a newly converted GIC population from differentiated GBM cells in vitro and in vivo after long-term exposure to TMZ (7). Therefore, it is vital to develop strategies to enhance the efficacy of TMZ in treating GICs (8).

Resveratrol (RES) is a natural polyphenolic compound that is widely present in plants and is enriched in red wine, peanuts, and grapes. It has exhibited a broad range of chemopreventive and therapeutic properties in a variety of animal models $(9,10)$. As a potential candidate for treating cancer, RES presents low toxicity and few adverse effects upon administration at relatively high doses (11). It has been shown that RES alters multiple signaling pathways, such as the JAK/ STAT, NF- $\kappa B / p 50 / p 65$ and p53 pathways, to induce cell cycle arrest, apoptosis and autophagy in various types of tumor cells (12-14). Specifically, RES potentiates the toxicity of TMZ in GBM cell lines such as SHG44 and T98 $(15,16)$. However, to the best of our knowledge, little is known regarding the effect of TMZ combined with RES on treating GICs which have distinct properties when compared to GBM cell lines. 
The aim of the present study was to investigate whether RES could enhance the antitumor effect of TMZ on GICs both in vitro and in vivo, and the involved mechanisms in response to the enhanced effects.

\section{Materials and methods}

GIC culture and cell immunofluorescence. GICs were derived from neurosurgical samples of two GBM patients at the Department of Neurosurgery, Beijing Tiantan Hospital, and informed consent was obtained from the patients. The use of human tissue specimens had been approved by the ethics board in our hospital. The tumor tissues were dissociated into single cells according to a previous study (6). To induce differentiation, the GICs were cultured in DMEM/F12 medium containing $10 \%$ fetal bovine serum (FBS) for two weeks. GICs and differentiated cells were immunofluorescence-stained with CD133, nestin, GFAP, NF, and CNP. The cell nuclei were stained with DAPI (Sigma-Aldrich, USA).

Limiting dilution assay. A limiting dilution assay was used to indicate the number of cells from a primary neurosphere (NS) that was needed to form a secondary NS. The specific method was described in a previous study (17).

Determination of GICs in NOD/SCID mice. The GICs ( $2 \times 10^{4} /$ mouse, 6 mice) were injected stereotactically into the right corpus striatum $(2.5 \mathrm{~mm}$ anterior and $2.5 \mathrm{~mm}$ lateral to the bregma and 3.0-mm deep) of 6-week-old female NOD/SCID mice (VitalStar, China). Once the mice were incapable, the brains were harvested. The brains were stained with hematoxylin and eosin (H\&E), nestin (GTX39578), and glial acidic fibrillary protein (GFAP, GTX84438) (both from GeneTex). All animal procedures were performed in accordance with the National Institutes of Health Guide for the Care and Use of Laboratory Animals.

Cell viability, apoptosis and sphere counting assay. MTT (Sigma-Aldrich, USA) assay was used to evaluate cell viability. The calculation of the combination index value (CI) was based on a previous study (18). Synergism, addition, and antagonism were defined as $\mathrm{CI}<1, \mathrm{CI}=1$, and $\mathrm{CI}>1$, respectively. To analyze apoptosis, the Annexin V/FITC and PI apoptosis detection kit was used according to the instructions provided by the manufacturer (Becton-Dickinson, USA). Quantification of apoptotic cells was performed using a FACScan flow cytometer. For the sphere counting assay which evaluates the self-renewal ability of GICs, colonies ( $>50 \mu \mathrm{m}$ in diameter) were counted after GICs were exposed to drugs for 2 weeks, and the number of colonies/number of cells in each well was calculated according to a previous study (19) with minor revisions. Each assay was repeated in three independent experiments.

Measurement of caspase-3 activity. Caspase-3 activity was assayed spectrophotometrically via the detection of pNA cleavage from caspase-3-specific substrates (Ac-LEVD-pNA) according to a commercially available kit (Beyotime, China).

DNA double-strand break (DSB) assay. DSBs were confirmed according to the OxiSelect ${ }^{\mathrm{TM}}$ DSB staining kit protocol. GICs exposed to etoposide at $100 \mu \mathrm{M}$ were regarded as the positive control. Cell images were acquired and analyzed under a Zeiss fluorescence microscope.

Western blot analysis. The cells were lysed in buffer (Cell Signaling Technology) containing $100 \mathrm{mM} \mathrm{NaF}$, and 1:500 protease inhibitor mixture (Roche, USA). Equal amounts of proteins were separated by 7-15\% SDS-PAGE for electrophoresis. The protein was hybridized by overnight incubation with the primary antibodies. ChemiDoc XRS+ image analyzer (Bio-Rad, USA) and ImageJ software (http://rsb.info.nih. gov/ij) were used to quantify the protein band density.

In vivo xenograft study and immunohistochemical study. GICs $\left(2 \times 10^{5}\right)$ were subcutaneously injected into the left hind flank of the 6-week-old female NOD/SCID mice. When the tumors grew to the 10th day, TMZ administration was began by oral gavage at doses of $68 \mathrm{mg} / \mathrm{kg}$, which corresponded to the murine equivalent of the standard clinical dose of $200 \mathrm{mg} /$ $\mathrm{m}^{2}$ and schedule as used by Yuan et al (15). TMZ was used for 5 days and halted for 10 days, and then repeated again. Meanwhile, $12.5 \mathrm{mg} / \mathrm{kg}$ RES was injected intraperitoneally once a day. Animal body weight, hair color and appearance were assessed every 5 days. The tumor sizes were measured every 5 days with a caliper and were calculated as $1 / 2 \times$ length $x$ width $^{2}$ in $\mathrm{mm}^{3}$. At day 40 of the inoculation, the mice were sacrificed and tumor tissues were excised for GFAP and nestin immunohistochemical study. Nestin-positive cells and GFAP-positive cells were counted in 5 staining fields chosen randomly.

Statistical analysis. The data are presented as the means \pm SD, and the Student's t-test was used for comparing paired sample sets. A P-value $<0.05$ was considered to indicate a statistically significant result.

\section{Results}

Determination of GICs in vitro and in vivo. Using the methods described above, we successfully isolated two patient-derived GIC cell lines and named them GIC400 and GIC411, respectively. The representative results of GIC400 determination are shown in Fig. 1 (similar results were also determined for GIC411; data not shown). As shown in Fig. 1A, these cells formed characteristic renewable neurospheres and could proliferate indefinitely, and all the GICs exhibited a high expression of nestin which is a neural progenitor cell marker while a large portion of the cells were stained positive for CD133 by immunofluorescence. Furthermore, upon serum exposure, GICs acquired the glial- and neurite-like cell features with protrusions and adherence to the flask under optical microscope, and the GICs showed a GFAP (astrocyte)-, NF (neuron)-, and CNP (oligodendrocyte)-directed differentiation morphology (Fig. 1B). The results were consistent with previous literature (17), showing that GICs could be efficiently induced into astrocytic, neural and partly induced into oligodendrocytic lineages after incubation with serum-containing DMEM/F12 media for 2 weeks. We then performed limiting dilution assays to confirm the enrichment of GICs in the primary cultures. GIC400 and GIC411 cells required a small number 

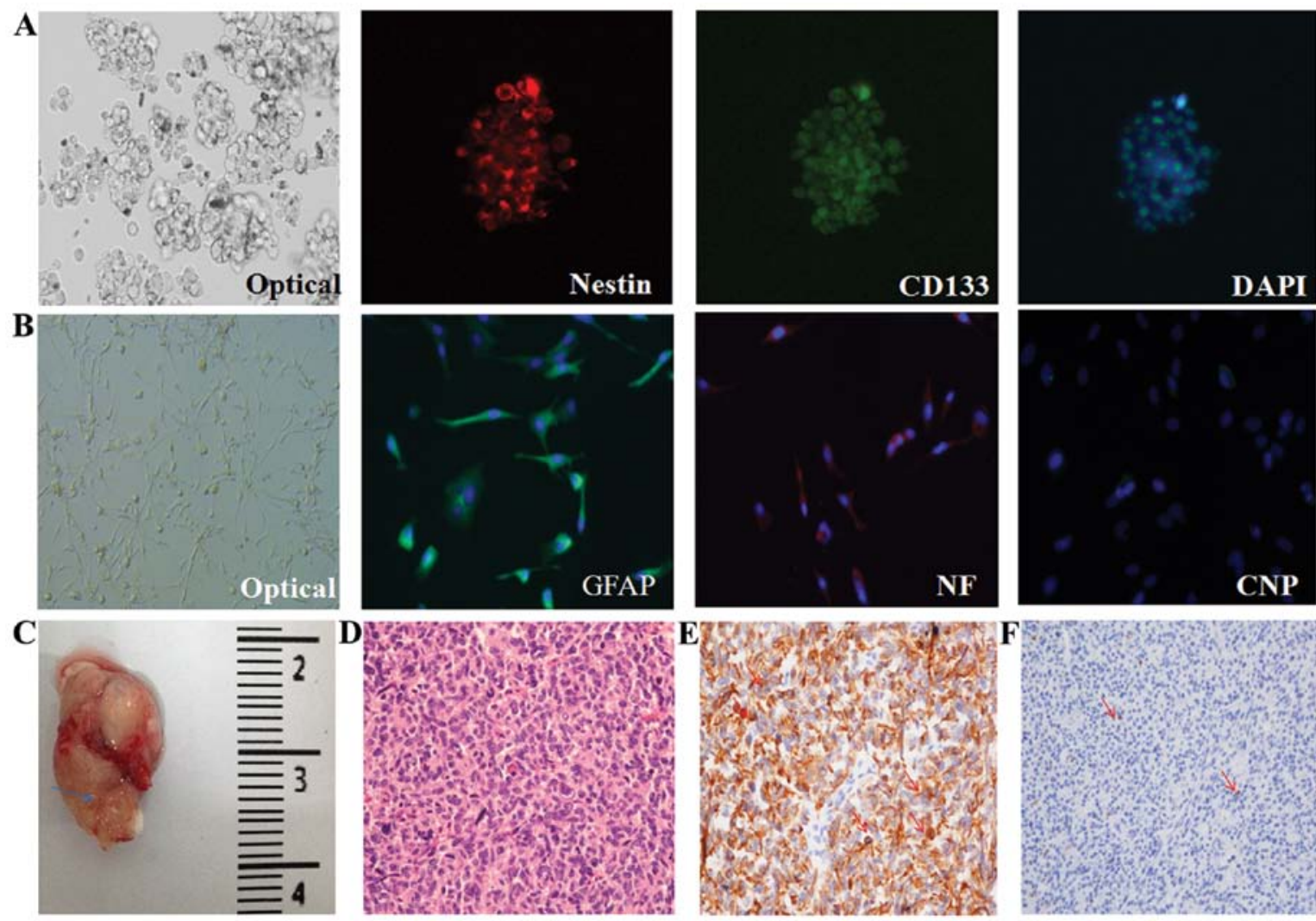

Figure 1. Determination of GICs in vitro and in vivo. (A) Morphology of tumor spheres in stem cell media (x40 magnification) and expression of stem cell markers (nestin, CD133) by immunostaining (x100 magnification). DAPI staining was used to indicate the location of GICs. (B) Serum-induced differentiation of GICs under a microscope (x40 magnification) and immunostaining of GFAP, NF and CNP (x200 magnification) expressed in differentiated GICs. (C) Tumorigenesis of GICs in the brain of NOD/SCID mice as indicated by the blue arrow, and H\&E staining (D) and nestin (E) and GFAP (F) immunostaining of brain tumors derived from GICs; the arrow indicates the positive staining site.

of cells to generate a secondary neurosphere (7.6 cells for GIC400, and 10.6 for GIC411) (data not shown). Furthermore, the potential for tumorigenesis was determined through intracranial tumor formation in the NOD/SCID mice for 2 months after inoculation with only $2 \times 10^{4}$ GICs (Fig. 1C), while GBM tumorigenesis generally requires $10^{6}$ non-GICs such as U87 cells. The tumors histologically resembled the GBM in patients through H\&E staining with the aid of a qualified expert pathologist in the brain tumor field, suggesting the successful establishment of the xenograft model of GICs according to the literature (20) (Fig. 1D). The xenograft samples also presented high expression levels of nestin (Fig. 1E) and acicular expression of GFAP (Fig. 1F), implying that the xenograft samples contained a high abundance of GICs.

RES has a synergistic effect with TMZ on GIC viability. The cell viability of GIC400 and GIC411 cells to TMZ, RES and the drug combination was evaluated after 6 days of exposure (Fig. 2A). The GIC400 cell line exhibited high resistance to TMZ while the half maximal inhibitory concentration $\left(\mathrm{IC}_{50}\right)$ of TMZ was $578 \mu \mathrm{M}$ and the $\mathrm{IC}_{50}$ of RES was $83 \mu \mathrm{M}$. Compared with the GIC400 cell line, the GIC411 cell line showed moderate resistant to $\mathrm{TMZ}$; the $\mathrm{IC}_{50}$ of $\mathrm{TMZ}$ was $331 \mu \mathrm{M}$. To evaluate whether RES could enhance the TMZ cytotoxicity on cell viability, we chose RES concentrations of 20 and $40 \mu \mathrm{M}$ (denoted R20 and R40 in short) and TMZ concentrations of 200 and $400 \mu \mathrm{M}$ (denoted T200 and T400 in short) for the combination treatment due to their moderate toxicity toward GICs when used alone (Fig. 2B and C). Synergistic effects on GIC400 and GIC411 cell viability were observed with the combined usage of R20 and T200 (CI $=0.88$ for GIC400), R20 and T400 (CI=0.72 for GIC400; $\mathrm{CI}=0.94$ for GIC411), R40 and T200 (CI=0.9 for GIC400; $\mathrm{CI}=0.93$ for GIC411), and R40 and T400 (CI=0.74 for GIC400; CI=0.9 for GIC411). In addition, an additive effect was observed in the combination of R20 and T200 for GIC411 cells (CI=1.03). Thus, we chose GIC400 cells for the following studies due to their highly resistant property to TMZ and a more obvious synergism of drug combination observed.

RES enhances TMZ-induced apoptosis of GICs via activation of the DSBs/pATM/pATR/p53 pathway. The treatment is shown in Fig. 2A, and the representative results of apoptosis are demonstrated in Fig. 3A. The percentage of total apoptosis of GICs was $20 \%$ when exposed to T200 and increased to $\sim 30 \%$ when exposed to T400. Combined with RES, TMZ induced the apoptosis significantly (the percentage of apoptosis was $34.5 \%$ when T200 was combined with R20, 39\% when T200 was combined with R40, 41.7\% when T400 was combined with R20, 50.8\% when T400 combined with R40).

The enhancement of apoptosis was evidenced by the elevation of caspase-3 activity. TMZ-treated GICs presented $\sim 2$ - to 3-fold higher caspase-3 activity than the control $(\mathrm{P}<0.05)$. A 4- to 9-fold increase in caspase-3 activity compared with the 
A

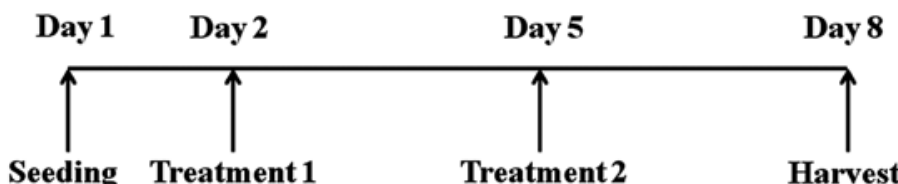

B
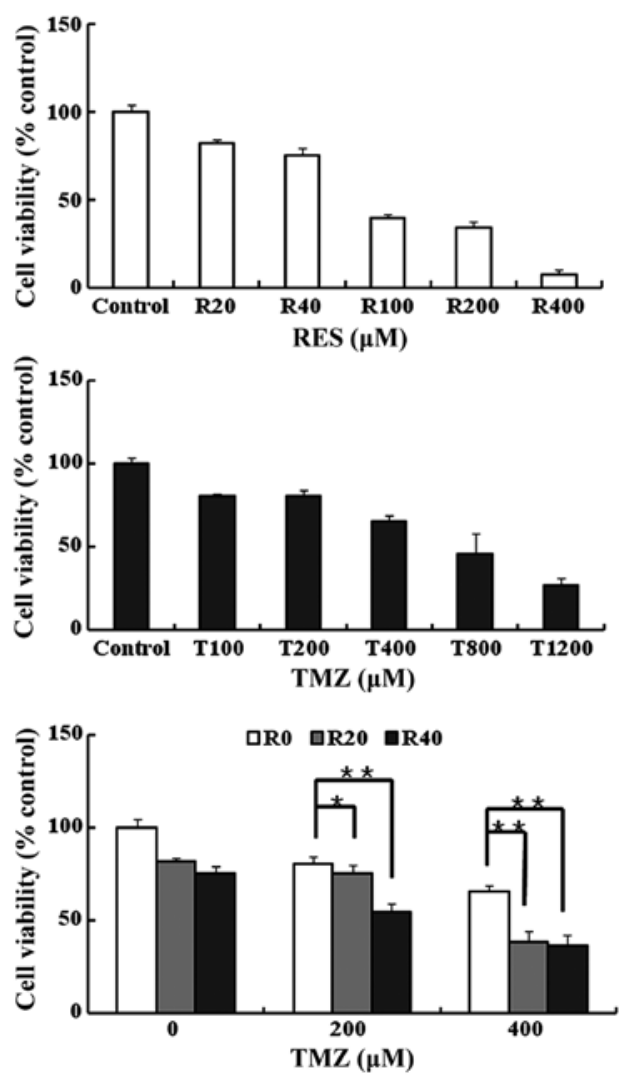

C
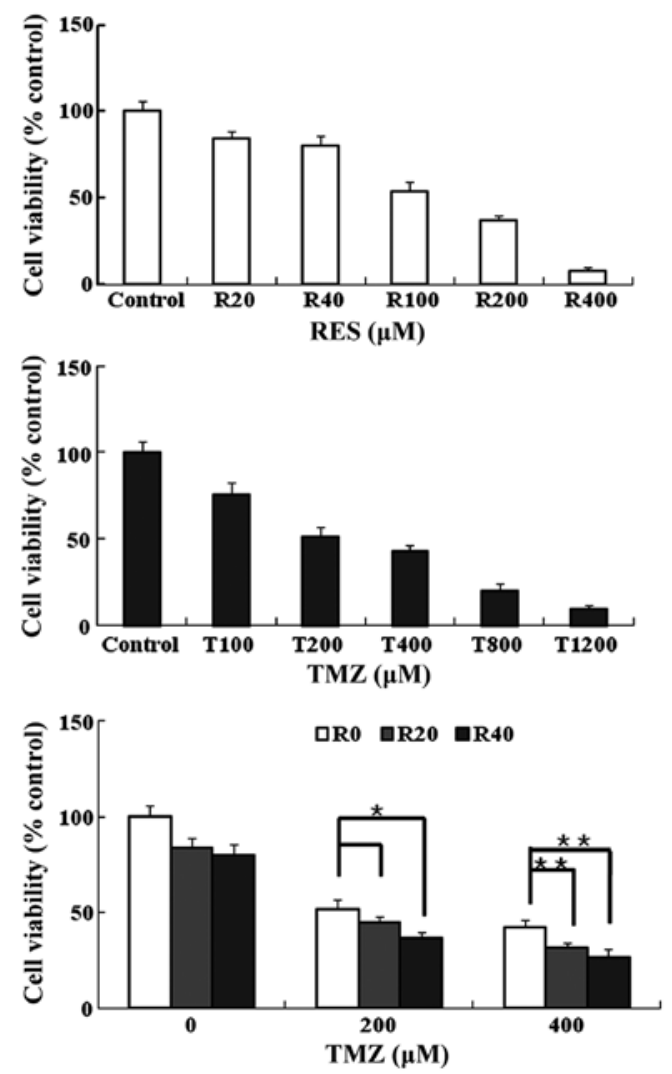

Figure 2. Inhibition of the viability of GICs by TMZ, RES and their combinations in vitro. (A) Drug treatment protocol for assessment of cell viability and apoptosis. (B) According to the treatment protocol as shown in Fig. 2A, the inhibitory effects of TMZ and RES alone or in combinations on cell viability of GIC400 cells for 6 days were determined using the MTT assay. (C) Inhibition of GIC411 cell viability by TMZ, RES and their combinations was determined by MTT assay. ${ }^{*} \mathrm{P}<0.05,{ }^{* *} \mathrm{P}<0.01$; error bars indicate standard error.

control was observed in the groups treated with the TMZ and RES combinations (Fig. 3B).

The presence of DSBs is determined by the elevation of phosphorylated histone $\mathrm{H} 2 \mathrm{AX}(\gamma \mathrm{H} 2 \mathrm{AX})$, which are DNA-damage downstream effectors (21). A marked increase in $\gamma \mathrm{H} 2 \mathrm{AX}$ expression was observed in the GICs exposed to R20 and R40, whereas a moderate increase was detected in the GICs exposed to T200 and T400. Furthermore, a significant increase in $\gamma \mathrm{H} 2 \mathrm{AX}$ expression was observed in the GICs exposed to the combination treatment with TMZ and RES (especially for R40 and T400) compared with that obtained with each agent alone (Fig. 3C and D).

$\mathrm{Ku} 70$ and MGMT which can repair DSBs and protect cells from death $(22,23)$, were moderately reduced in the GICs exposed to R20 and R40 in a dose-dependent manner, while the expression remained unchanged when GICs were exposed to TMZ alone except T400 (T400 inhibited MGMT expression). In addition, PARP1, another DSB repair protein, was decreased following treatment with T400 rather than with R20, R40 and T200. However, Ku70, MGMT and PARP1 were markedly reduced in the GICs exposed to the drug combinations compared with the levels obtained with each agent alone (Fig. 3E).

Important sensors of DSBs are ATM (ataxia telangiectasia mutated) and ATR (ataxia telangiectasia and Rad3 related) proteins, which signal downstream to p53 (24). The level of pATM was moderately upregulated while pATR, p53 and pp53 were significantly increased when TMZ was combined with RES. The representative graphics are shown in Fig. 3F. Moreover, the ratio of $\mathrm{Bcl}-2$ to $\mathrm{Bax}$, which is regulated by $\mathrm{p} 53$ expression, was significantly reduced in the GICs exposed to the combination treatment with TMZ and RES than the control $(\mathrm{P}<0.01)$ (Fig. 3F and $\mathrm{G})$.

Inhibition of self-renewal capacity and induction of cell differentiation via STAT3 inactivation in GICs exposed to the combinations with TMZ and RES. As demonstrated in Fig. 4A, the sphere-formation ability of GICs was slightly affected by $\mathrm{T} 200$, but a $\sim 50 \%$ reduction in this ability was induced by $\mathrm{T} 400(\mathrm{P}<0.01, \mathrm{~T} 400$ vs. control). Combined with 
A

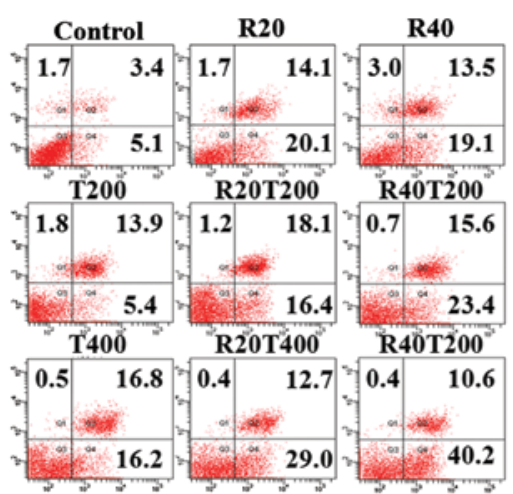

C
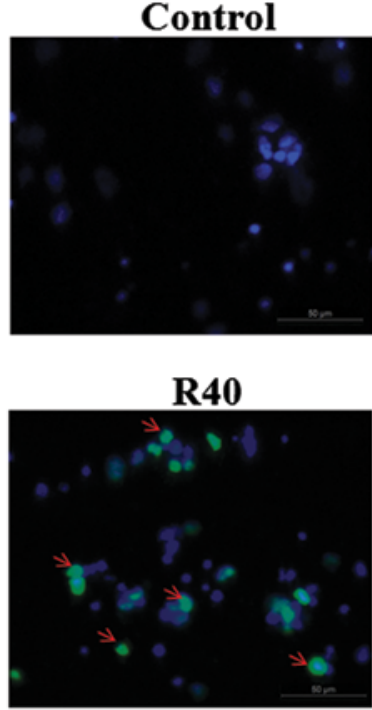

$\mathbf{E}$

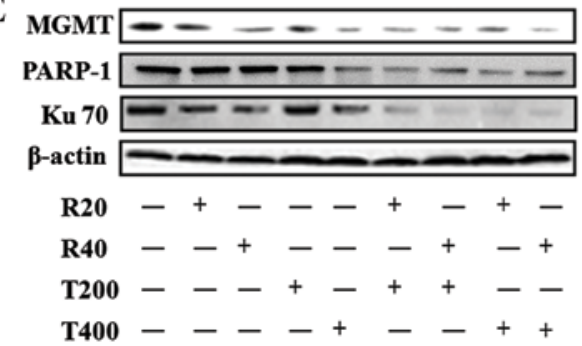

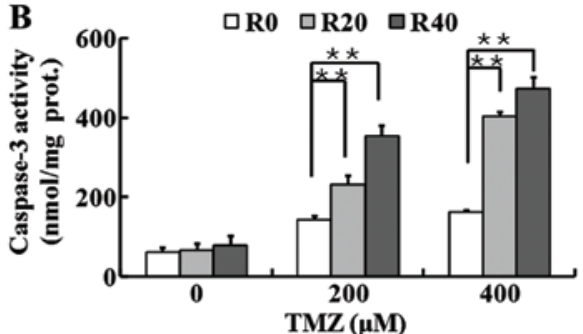

D

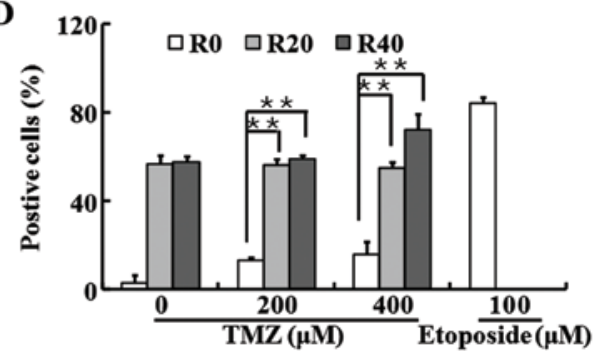

F

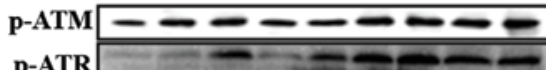

p53

p53pSer15 $\ldots-\ldots$

Bcl-2

Bax $-\cdots-\cdots$

p-actin

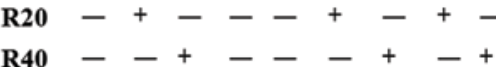

T200 - - + -++--

T400 - - - + - +

G

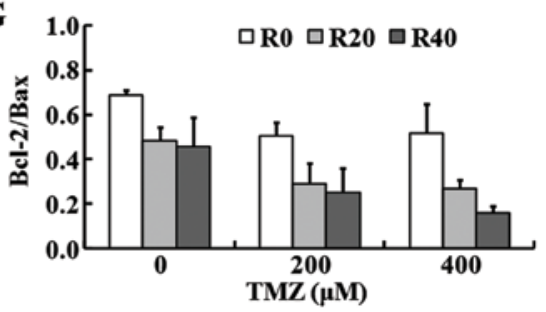

Figure 3. Cell apoptosis was induced in GICs and the DSB response was increased upon exposure to TMZ, RES and their combined usage. (A) Representative graphs of the flow cytometric analysis and quantification of cell apoptosis by Annexin V/PI staining. (B) The caspase-3 activity was assayed spectrophotometrically via the detection of pNA cleavage from caspase-3-specific substrates (Ac-LEVD-pNA). (C) Representative images of $\gamma \mathrm{H} 2 \mathrm{AX}$ expression. The arrow indicates positive cells. (D) Quantifications of $\gamma \mathrm{H} 2 \mathrm{AX}$ expression ( $\mathrm{n}=5$ ). (E) Representative images of the western blot analysis of the DNA damage repair proteins MGMT, PARP1 and Ku70 in GICs exposed to TMZ and RES combinations or each agent alone. (F) Representative images of the western blot analysis of the DSB response proteins pATM, pATR, p53, p53pSer15, Bcl-2 and Bax in GICs exposed to the TMZ and RES combinations or each agent alone. (G) Data from three independent experiments used for the semi-quantification of $\mathrm{Bcl}-2 / \mathrm{Bax}$ in GICs are expressed as the means $\pm \mathrm{SD}$. ${ }^{*} \mathrm{P}<0.05,{ }^{* *} \mathrm{P}<0.01$; error bars indicate standard error.

RES, the TMZ treatments significantly impaired the capacity of GICs to form spheres, similar to the effects observed in the GICs exposed to RES rather than TMZ (Fig. 4A).

Moreover, slight changes in the expression levels of CD133, nestin and GFAP were observed in the GICs exposed to T200 and T400. When involved by RES, the drug combinations significantly increased GFAP expression levels and decreased CD133 and nestin levels.

pSTAT3 (Y705) rather than pSTAT3 (S727) and the total STAT3 was found to be significantly suppressed when the self-renewal capacity was lost and differentiation was promoted in the GICs (Fig. 4C).
RES enhances TMZ-induced inhibition of the tumor growth in a xenograft model of GICs and promotes differentiation. To translate our findings into a clinically relevant approach, the drug combination effects were determined in vivo. Compared with an intracranial xenograft model, a subcutaneous xenograft model of GICs makes it easier to observe the tumor growth rate and measure tumor volume. Therefore, we adopted a subcutaneous xenograft model as described in a previous study (25). The results (Fig. 5A) showed that $68 \mathrm{mg} / \mathrm{kg}$ TMZ treatment alone exhibited an antitumor effect in regards to tumor volume growth inhibition $(\mathrm{P}=0.013$, TMZ vs. control at day 40). RES treatment alone did not significantly inhibit 
A

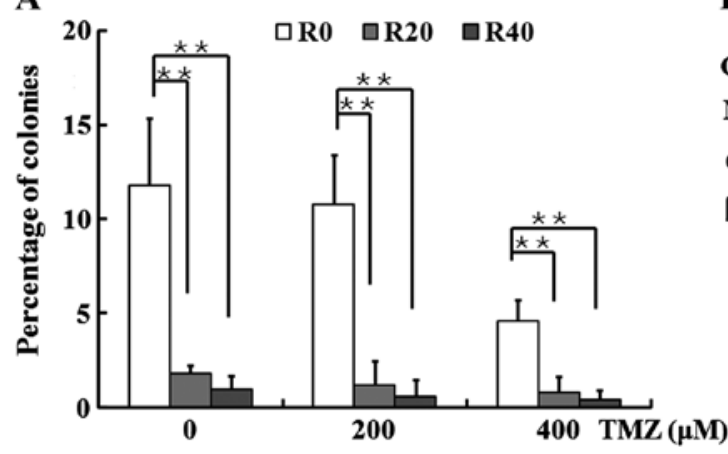

C

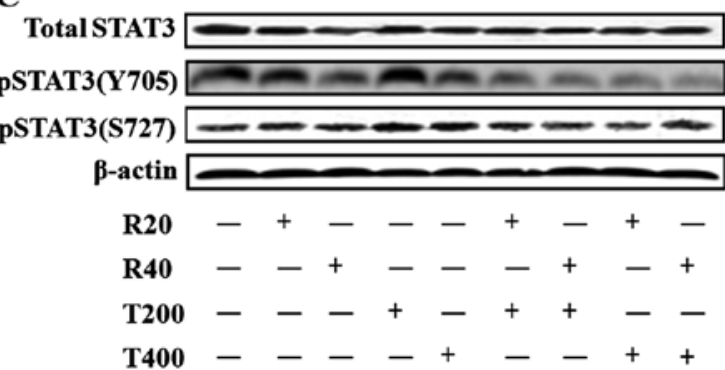

B

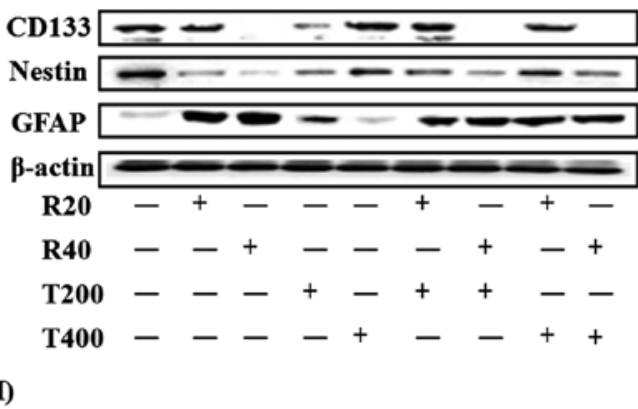

Figure 4. Inhibition of sphere-formation ability and induction of cell differentiation in GICs exposed to the combined treatments of TMZ and RES. (A) Assay of the sphere-formation ability of GICs exposed to the combined treatments of TMZ and RES, or each agent alone ( $\mathrm{n}=6$ ). ${ }^{*} \mathrm{P}<0.05$, ** $\mathrm{P}<0.01$; error bars indicate standard error. (B) Representative images of the western blot analysis of CD133, nestin, GFAP, and $\beta$-actin in GICs exposed to the combined treatments of TMZ and RES, or each agent alone. (C) Representative images of the western blot analysis of total STAT3, pSTAT3(Y705), pSTAT3(S727) and $\beta$-actin in GICs exposed to the combined treatments of TMZ and RES, or each agent alone. Data are representative of three independent experiments.

the growth of the tumors at the dose of $12.5 \mathrm{mg} / \mathrm{kg}(\mathrm{P}=0.113$ vs. control at day 40). RES significantly potentiated TMZ in inhibiting tumor volume growth $(\mathrm{P}=0.035$, TMZ+RES vs. TMZ). As shown in Fig. 5B, the combination of $68 \mathrm{mg} / \mathrm{kg}$ $\mathrm{TMZ}$ and $12.5 \mathrm{mg} / \mathrm{kg}$ RES significantly reduced the excised tumor volume by $77.1 \%$ compared with that observed in the control group ( $\mathrm{P}=0.034$ vs. control). Animal body weight was monitored serially to assess the tolerability of the regimens tested in all mice (data not shown). In the course of treatment, there was no significant difference in body weight between the control group and RES group. Moreover, there was no significant increase or decrease in the average mouse weight in the TMZ alone and TMZ+RES groups between days 0 and 40 , with the exception of day 15 , where a decrease in body weight was observed. However, the body weight was $15 \%$ and $17 \%$ lower compared with the control group in the TMZ and TMZ+RES groups, respectively. Of note, there were no deaths during the treatment course. The results indicated that the combination therapy did not have increased toxicity compared to TMZ alone.

Moreover, as shown in Fig. 5C, the density of the nestin-immunoreactive 'stemness' cell surface in the TMZ group was similar to that in the control group $(\mathrm{P}=0.15)$. TMZ combined with RES robustly downregulated nestin expression by $58.5 \%$ compared with the control group $(\mathrm{P}=0.005 \mathrm{TMZ}+\mathrm{RES}$ vs. control). GFAP immunostaining was rare in the control group and the TMZ group; treatment with RES combined with TMZ greatly increased the GFAP-immunoreactive astrocytic surface density by $116.1 \%$ ( $\mathrm{P}=0.0009, \mathrm{TMZ}+\mathrm{RES}$ vs. control) and $57.9 \%(\mathrm{P}=0.002$, TMZ+RES vs. TMZ).

\section{Discussion}

Using highly resistant GICs isolated from patient samples, the present study demonstrated that RES enhanced the sensitivity of TMZ by inducing the apoptosis of GICs via activation of the DSBs/pATM/pATR/p53 pathway. Moreover, the involvement of RES in TMZ therapy also reduced the self-renewal ability and promoted the differentiation of GICs by the inactivation of pSTAT3.

RES enhances TMZ-induced GIC apoptosis via activation of the DSBs/pATM/pATR/p53 pathway. A recent study demonstrated that RES potentiated the efficacy of TMZ by suppressing TMZ-induced autophagy and subsequently increasing apoptotic cell death in glioma cell lines (26). However, whether RES enhances the TMZ-induced apoptosis in GICs from glioma cell lines due to their distinct properties and the mechanism underlying the enhanced apoptosis induced by TMZ and RES combination has not yet been reported.

In the present study, we found that RES significantly enhanced TMZ-induced apoptosis of GICs. As an alkylating agent, TMZ induces the $\mathrm{O}^{6}$-methylguanine lesion which leads to DSBs via collapse of a replication fork at the site of a blocking DNA lesion and results in cell death via apoptosis and/or autophagy (27). In addition, several studies have shown that RES poisons TOPOIIa thus inducing DSBs and activating the DSB signaling pathway to induce apoptosis (28). Given that TMZ or RES alone leads to tumor cell apoptosis through DSB formation capacity, we found that their combinations significantly induced DSBs underlying apoptosis, evidenced by 
A

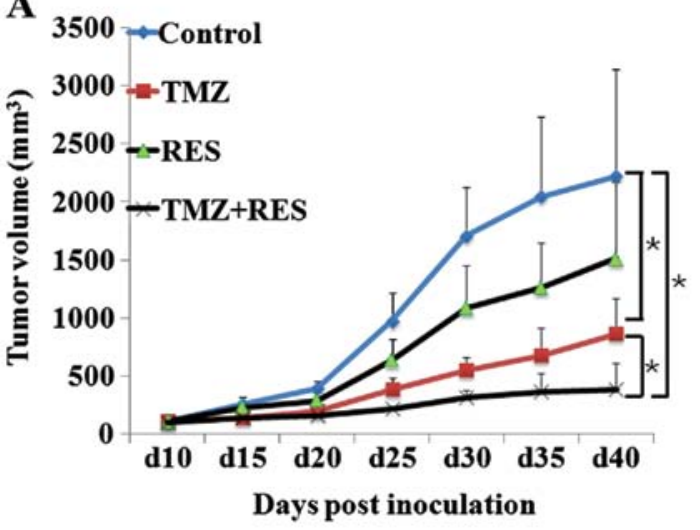

B

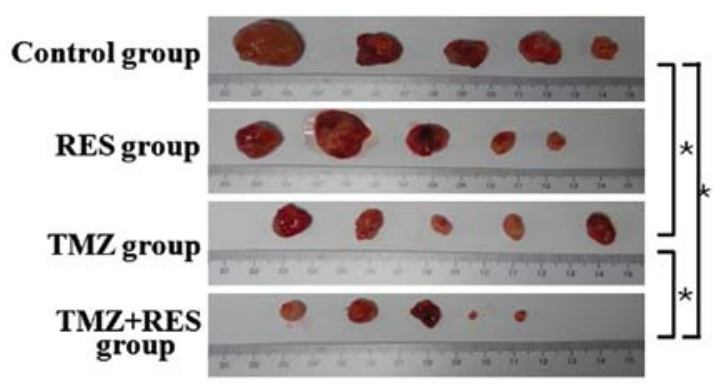

C
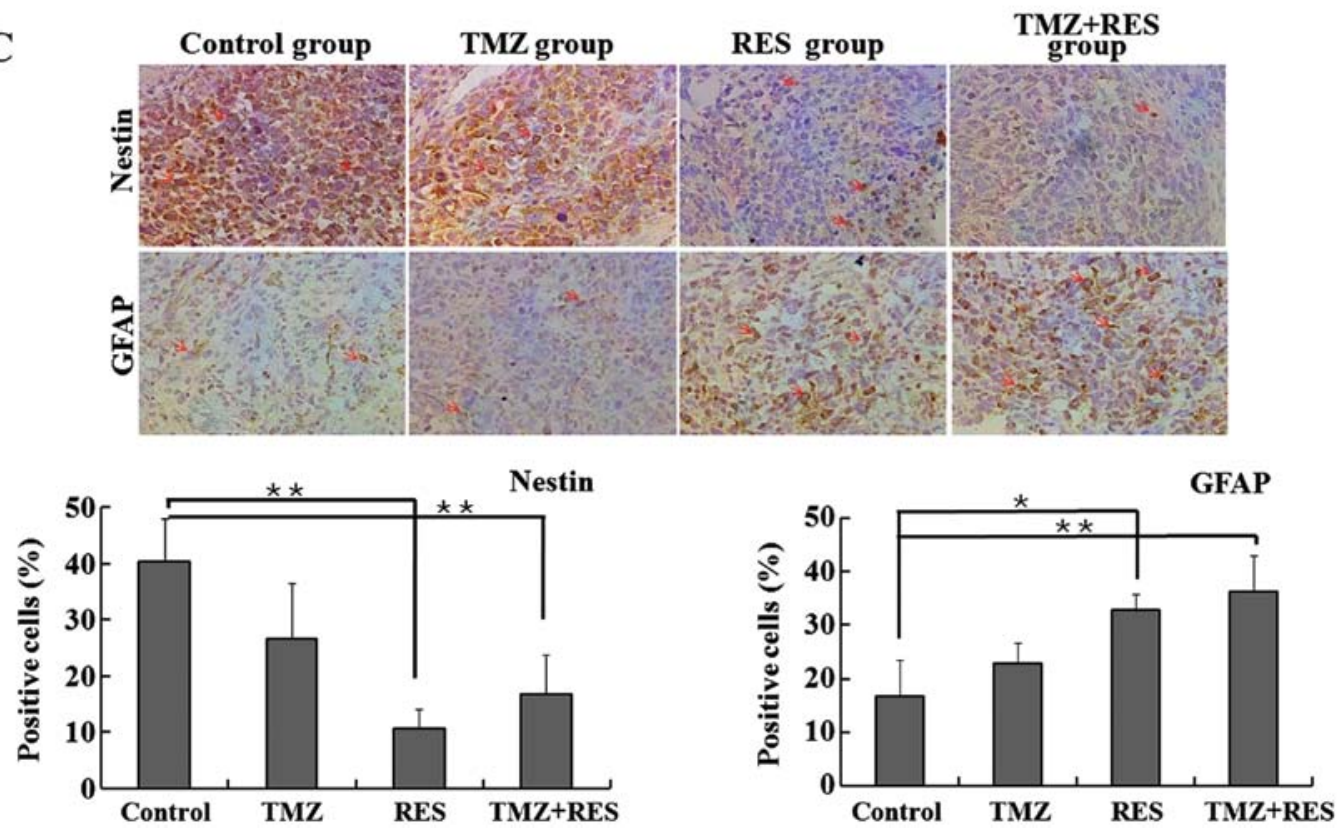

Figure 5. Resveratrol enhances the efficacy of TMZ and promotes differentiation in a GIC xenograft model. (A) Mice with GIC xenografts were injected intraperitoneally with $12.5 \mathrm{mg} / \mathrm{kg}$ resveratrol daily, TMZ (68 mg/kg by oral gavage for 5 days and halted for 10 days followed by another round of TMZ administration for 5 days and halted for 10 days) and their combination from day 10 to day 40 . Tumor length and width were measured every 5 days. (B) Images of excised tumors after the mice were sacrificed. The tumor length and width were measured and the tumor volume was calculated as $1 / 2 \mathrm{x}$ length $\mathrm{x}$ width ${ }^{2}$ in $\mathrm{mm}^{3}$. (C) Representative images and statistical analysis of the immunohistochemical analysis of nestin and GFAP expression in the GIC xenograft model. The arrow indicates the positive cells. ${ }^{*} \mathrm{P}<0.05,{ }^{* *} \mathrm{P}<0.01$; error bars indicate standard error.

$\gamma \mathrm{H} 2 \mathrm{AX}$ focus formation. Furthermore, it has been suggested that DNA damage repair proteins may prevent DSBs and protect tumor cells from death and they are considered as important determinants of chemoresistance in many tumors including GBM (29,30). Thus, we assessed the expression levels of three DNA damage repair proteins MGMT, PARP1 and Ku70 due to their important roles in removing DNA lesions, alternative end-joining (A-EJ) repair and the non-homologous end joining (NHEJ) repair mechanism, respectively $(22,31,32)$. Our results showed that combined usage of TMZ and RES markedly repressed MGMT, PARP1 and Ku70 compared with that obtained with either chemical alone, suggesting that the DSB repair system was broadly inhibited.

The signaling pathway orchestrated by the ATM and ATR kinases is the central regulator in bridging DSBs to final apoptosis $(24,33)$. Although ATM and ATR often work together to signal DSBs, ATM is primarily activated through DSBs caused by extrinsic stress such as irradiation and chemical toxicity while ATR is considered mainly activated in response to replication stress (34). Compared with slight increase in pATM expression, there was a significant upregulation of pATR when TMZ was combined with RES in our study. This implies that p-ATR activation principally functions as the bridge to pass down the DSB signal to downstream effectors, and replication stress might be mainly responsible for DSB formation induced by the current concentrations of TMZ, RES and their combinations.

p53 contributes significantly to the maintenance of genomic stability and is the core component of the network in regulating apoptosis (35). ATM and ATR could both directly phosphorylate p53 to activate and stabilize the protein. Thus, the DSBs/pATM/pATR/p53 signaling pathway has been widely reported to play key roles in regulating apoptosis induced by DNA damage in previous studies $(36,37)$. In accordance with these results, we found that p53 and its phosphorylated form were highly elevated accompanying the activation of pATM/ 
pATR in the use of drug combinations. Furthermore, due to the role of 553 activation in the regulation of Bcl-2/Bax both transcriptionally and at the protein level (38), we also demonstrated that the $\mathrm{Bcl}-2 / \mathrm{Bax}$ ratio was significantly decreased when TMZ was combined with RES.

The involvement of RES in TMZ treatment reduces the self-renewal ability and promotes differentiation of GICs with the inactivation of pSTAT3. The 'stemness' of GICs is commonly believed to be the significant property accounting for therapeutic resistance and the capability of repopulation for GBM (39). In our study, GICs exposed to TMZ exhibited no change in their 'stemness' phenotype as shown in Fig. 4B, which is in line with previous studies $(6,7)$. The suppression of sphere-formation ability of GICs by TMZ may be due to increases in apoptosis and cell cycle arrest (data not shown) induced by DNA damage.

Resveratrol was previously found to promote the acquisition of a long-lasting differentiated phenotype in human GBM cells and to inhibit the tumorigenicity of GICs (40). In our study, a marked decreased tendency of GIC expansion was observed when TMZ was combined with RES as these cells lost their self-renewal capacity and underwent a conversion from 'stemness' to a differentiation phenotype, evidenced by decreases in the expression levels of CD133 and nestin and an increase in the expression of GFAP.

STAT3 is important in maintaining the self-renewal of GICs and the inactivation of STAT3 in GICs is identified as the onset signal for the conversion from a 'stemness' phenotype to a differentiation phenotype (19,41-43). For instance, suppression of STAT3 with siRNA significantly induced GIC differentiation with a decrease in CD133, increase in GFAP and a decrease in capacity for GICs to initiated a tumor. STAT3 is activated mainly by phosphorylation at Tyr705, which induces dimerization, nuclear translocation, and DNA binding. In addition, phosphorylation of STAT3 at Ser727 is usually considered to be necessary for the maximal transcriptional activity $(44,45)$. Moreover, constitutive activation of STAT3 at both Tyr705 and Ser727 has been observed in many human malignancies including GBM (46). In the present study, we found that STAT3-Tyr705 was significantly suppressed when GICs were converting from a 'stemness' phenotype to a differentiation status while STAT3-Ser727 and STAT3 remained almost the same, suggesting that STAT3-Tyr705 suppression by RES and drug combinations plays a key role in inhibition of self-renewal ability and promotion of differentiation.

In summary, the present study introduces evidence that RES may act in concert with TMZ to eradicate GICs, thereby providing a foundation for the combined usage of RES and TMZ on GBM patients, particularly those with abundant GICs in their histopathological sections. This new strategy targeting GICs may benefit patients who show slight responsiveness to TMZ therapy and provide a promising long-term survival effect compared with TMZ therapy alone.

\section{Acknowledgements}

The authors would like to thank Dr Pingyang Liu at the University of California, San Francisco for critically reading and discussing this manuscript. This study was supported by a grant from the National Natural Science Foundation of China (81102463), funds from the China National Clinical Research Center for Neurological Diseases, the Training Plan for Beijing High-Level Healthcare Personnel (2011-3-28), and funds of the Capital Medical University Clinical-Basic Cooperation Research (11JL16).

\section{References}

1. Cheng L, Bao S and Rich JN: Potential therapeutic implications of cancer stem cells in glioblastoma. Biochem Pharmacol 80: 654-665, 2010.

2. Orza A, Soriţău O, Tomuleasa C, Olenic L, Florea A, Pana O, Bratu I, Pall E, Florian S, Casciano D, et al: Reversing chemoresistance of malignant glioma stem cells using gold nanoparticles. Int J Nanomedicine 8: 689-702, 2013.

3. Higgins DM, Wang R, Milligan B, Schroeder M, Carlson B, Pokorny J, Cheshier SH, Meyer FB, Weissman IL, Sarkaria JN, et al: Brain tumor stem cell multipotency correlates with nanog expression and extent of passaging in human glioblastoma xenografts. Oncotarget 4: 792-801, 2013.

4. Neman J and Jandial R: Decreasing glioma recurrence through adjuvant cancer stem cell inhibition. Biologics 4: 157-162, 2010.

5. Johannessen TC, Bjerkvig R and Tysnes BB: DNA repair and cancer stem-like cells - potential partners in glioma drug resistance? Cancer Treat Rev 34: 558-567, 2008.

6. Beier D, Schriefer B, Brawanski K, Hau P, Weis J, Schulz JB and Beier CP: Efficacy of clinically relevant temozolomide dosing schemes in glioblastoma cancer stem cell lines. J Neurooncol 109: 45-52, 2012.

7. Auffinger B, Tobias AL, Han Y, Lee G, Guo D, Dey M, Lesniak MS and Ahmed AU: Conversion of differentiated cancer cells into cancer stem-like cells in a glioblastoma model after primary chemotherapy. Cell Death Differ 21: 1119-1131, 2014.

8. Persano L, Rampazzo E, Basso G and Viola G: Glioblastoma cancer stem cells: Role of the microenvironment and therapeutic targeting. Biochem Pharmacol 85: 612-622, 2013.

9. Sales JM and Resurreccion AV: Resveratrol in peanuts. Crit Rev Food Sci Nutr 54: 734-770, 2014.

10. Borriello A, Bencivenga D, Caldarelli I, Tramontano A, Borgia A, Zappia V and Della Ragione F: Resveratrol: From basic studies to bedside. Cancer Treat Res 159: 167-184, 2014

11. Pallàs $\mathrm{M}$, Ortuño-Sahagún $\mathrm{D}$, Benito-Andrés $\mathrm{P}$, PonceRegalado MD and Rojas-Mayorquín AE: Resveratrol in epilepsy: Preventive or treatment opportunities? Front Biosci (Landmark Ed) 19: 1057-1064, 2014.

12. Aggarwal BB, Bhardwaj A, Aggarwal RS, Seeram NP Shishodia $S$ and Takada Y: Role of resveratrol in prevention and therapy of cancer: Preclinical and clinical studies. Anticancer Res 24: 2783-2840, 2004.

13. Harikumar KB and Aggarwal BB: Resveratrol: A multitargeted agent for age-associated chronic diseases. Cell Cycle 7: 1020-1035, 2008.

14. Delmas D, Solary E and Latruffe N: Resveratrol, a phytochemical inducer of multiple cell death pathways: Apoptosis, autophagy and mitotic catastrophe. Curr Med Chem 18: 1100-1121, 2011.

15. Yuan Y, Xue X, Guo RB, Sun XL and Hu G: Resveratrol enhances the antitumor effects of temozolomide in glioblastoma via ROS-dependent AMPK-TSC-mTOR signaling pathway. CNS Neurosci Ther 18: 536-546, 2012.

16. Huang H, Lin H, Zhang X and Li J: Resveratrol reverses temozolomide resistance by downregulation of MGMT in T98G glioblastoma cells by the NF- $\kappa \mathrm{B}$-dependent pathway. Oncol Rep 27: 2050-2056, 2012.

17. Aldaz B, Sagardoy A, Nogueira L, Guruceaga E, Grande L, Huse JT, Aznar MA, Díez-Valle R, Tejada-Solís S, Alonso MM, et al: Involvement of miRNAs in the differentiation of human glioblastoma multiforme stem-like cells. PLoS One 8: e77098, 2013.

18. Romanelli S, Perego P, Pratesi G, Carenini N, Tortoreto M and Zunino F: In vitro and in vivo interaction between cisplatin and topotecan in ovarian carcinoma systems. Cancer Chemother Pharmacol 41: 385-390, 1998.

19. Yang YP, Chang YL, Huang PI, Chiou GY, Tseng LM, Chiou SH, Chen MH, Chen MT, Shih YH, Chang CH, et al: Resveratrol suppresses tumorigenicity and enhances radiosensitivity in primary glioblastoma tumor initiating cells by inhibiting the STAT3 axis. J Cell Physiol 227: 976-993, 2012. 
20. Gedye $C$ and Ailles L: Isolation and characterization of cancer stem cells in vitro. Methods Mol Biol 946: 181-204, 2013.

21. Roos WP and Kaina B: DNA damage-induced cell death: From specific DNA lesions to the DNA damage response and apoptosis. Cancer Lett 332: 237-248, 2013.

22. Ponnala S, Veeravalli KK, Chetty C, Dinh DH and Rao JS: Regulation of DNA repair mechanism in human glioma xenograft cells both in vitro and in vivo in nude mice. PLoS One 6: e26191, 2011.

23. Silber JR, Bobola MS, Blank A and Chamberlain MC: $\mathrm{O}(6)$-methylguanine-DNA methyltransferase in glioma therapy: Promise and problems. Biochim Biophys Acta 1826: 71-82, 2012

24. Park I and Avraham HK: Cell cycle-dependent DNA damage signaling induced by ICRF-193 involves ATM, ATR, CHK2, and BRCA1. Exp Cell Res 312: 1996-2008, 2006.

25. Eyler CE, Wu Q, Yan K, MacSwords JM, Chandler-Militello D, Misuraca KL, Lathia JD, Forrester MT, Lee J, Stamler JS, et al: Glioma stem cell proliferation and tumor growth are promoted by nitric oxide synthase-2. Cell 146: 53-66, 2011

26. Lin CJ, Lee CC, Shih YL, Lin TY, Wang SH, Lin YF and Shih CM: Resveratrol enhances the therapeutic effect of temozolomide against malignant glioma in vitro and in vivo by inhibiting autophagy. Free Radic Biol Med 52: 377-391, 2012.

27. Johannessen TC and Bjerkvig R: Molecular mechanisms of temozolomide resistance in glioblastoma multiforme. Expert Rev Anticancer Ther 12: 635-642, 2012.

28. Leone S, Basso E, Polticelli F and Cozzi R: Resveratrol acts as a topoisomerase II poison in human glioma cells. Int J Cancer 131: E173-E178, 2012.

29. Srivastava $M$ and Raghavan SC: DNA double-strand break repair inhibitors as cancer therapeutics. Chem Biol 22: 17-29, 2015

30. Aparicio T, Baer R and Gautier J: DNA double-strand break repair pathway choice and cancer. DNA Repair (Amst) 19: 169-175, 2014.

31. Villalva C, Cortes U, Wager M, Tourani JM, Rivet P, Marquant C, Martin S, Turhan AG and Karayan-Tapon L: ${ }^{6}-$ Methylguanine-methyltransferase (MGMT) promoter methylation status in glioma stem-like cells is correlated to temozolomide sensitivity under differentiation-promoting conditions. Int $\mathrm{J} \mathrm{Mol}$ Sci 13: 6983-6994, 2012.

32. Haince JF, McDonald D, Rodrigue A, Déry U, Masson JY, Hendzel MJ and Poirier GG: PARP1-dependent kinetics of recruitment of MRE11 and NBS1 proteins to multiple DNA damage sites. J Biol Chem 283: 1197-1208, 2008

33. Gobbini E, Cesena D, Galbiati A, Lockhart A and Longhese MP Interplays between ATM/Tel1 and ATR/Mec1 in sensing and signaling DNA double-strand breaks. DNA Repair (Amst) 12: $791-799,2013$
34. Cooper TJ, Wardell K, Garcia V and Neale MJ: Homeostatic regulation of meiotic DSB formation by ATM/ATR. Exp Cell Res 329: 124-131, 2014

35. Speidel D: The role of DNA damage responses in p53 biology. Arch Toxicol 89: 501-517, 2015.

36. Shimada M and Nakanishi M: Response to DNA damage: Why do we need to focus on protein phosphatases? Front Oncol 3: 8 , 2013.

37. Loewer A, Karanam K, Mock C and Lahav G: The p53 response in single cells is linearly correlated to the number of DNA breaks without a distinct threshold. BMC Biol 11: 114, 2013.

38. Kolb JP: Mechanisms involved in the pro- and anti-apoptotic role of NO in human leukemia. Leukemia 14: 1685-1694, 2000.

39. Nakano I: Stem cell signature in glioblastoma: Therapeutic development for a moving target. J Neurosurg 122: 324-330, 2015.

40. Sato A, Okada M, Shibuya K, Watanabe E, Seino S, Suzuki K, Narita Y, Shibui S, Kayama T and Kitanaka C: Resveratrol promotes proteasome-dependent degradation of Nanog via p53 activation and induces differentiation of glioma stem cells. Stem Cell Res (Amst) 11: 601-610, 2013.

41. Li GH, Wei H, Lv SQ, Ji H and Wang DL: Knockdown of STAT3 expression by RNAi suppresses growth and induces apoptosis and differentiation in glioblastoma stem cells. Int J Oncol 37: 103-110, 2010.

42. Yang L, Guo H, Dong L, Wang L, Liu C and Wang X: Tanshinone IIA inhibits the growth, attenuates the stemness and induces the apoptosis of human glioma stem cells. Oncol Rep 32: 1303-1311, 2014

43. Liu M, Inoue K, Leng T, Guo S and Xiong ZG: TRPM7 channels regulate glioma stem cell through STAT3 and Notch signaling pathways. Cell Signal 26: 2773-2781, 2014.

44. Lin J, Jin X, Rothman K, Lin HJ, Tang H and Burke W: Modulation of signal transducer and activator of transcription 3 activities by p53 tumor suppressor in breast cancer cells. Cancer Res 62: 376-380, 2002.

45. Yang F, Zhang W, Li D and Zhan Q: Gadd45a suppresses tumor angiogenesis via inhibition of the mTOR/STAT3 protein pathway. J Biol Chem 288: 6552-6560, 2013.

46. Gray GK, McFarland BC, Nozell SE and Benveniste EN: NF- $\kappa B$ and STAT3 in glioblastoma: Therapeutic targets coming of age. Expert Rev Neurother 14: 1293-1306, 2014. 